\title{
Rotenone- and Cyanide-insensitive Respiration in Mitochondria from Neurospora crassa
}

\author{
By JEAN-PAUL SCHWITZGUÉBEL*† AND JOHN M. PALMER \\ Department of Pure and Applied Biology, Imperial College of Science and Technology, \\ Prince Consort Road, London SW7 2BB, U.K.
}

(Received 4 February 1983)

\begin{abstract}
When Neurospora crassa was grown in the presence of chloramphenicol, the oxidation of $\mathrm{NAD}^{+}-$ linked substrates by isolated mitochondria became largely insensitive to rotenone. It appears that chloramphenicol hindered the biogenesis of a functional rotenone-sensitive NADHubiquinone oxidoreductase. The rotenone-resistant pathway was able to donate electrons to both cyanide-sensitive and -resistant oxidases, indicating the operation of a branched system rather than of parallel respiratory chains. Growth of $N$. crassa in the presence of chloramphenicol also strongly enhanced the rates of oxidation of exogenous NADH and NADPH. This increased oxidation by mitochondria was largely insensitive to cyanide. The addition of exogenous AMP to isolated mitochondria did not specifically stimulate the electron flux through the alternative oxidase.
\end{abstract}

\section{INTRODUCTION}

The mitochondrial respiration of wild-type Neurospora crassa is mediated by a conventional cytochrome chain, sensitive to antimycin and cyanide (Lambowitz et al., 1972a, b). When protein synthesis on mitochondrial ribosomes is hindered by the addition of chloramphenicol to the growth medium (La Polla \& Lambowitz, 1977), respiration via the cytochrome chain is reduced, and an alternative oxidase is derepressed (Lambowitz et al., 1972c; Von Jagow \& Bohrer, 1975; Slayman, 1978). The antimycin- and cyanide-insensitive oxidase of $N$. crassa appears to be similar to the alternative oxidase described for other micro-organisms and several higher plants, since it is inhibited by substituted hydroxamic acids and is not linked to phosphorylation (De Troostembergh \& Nyns, 1978; Edwards \& Lloyd, 1978; Shepherd et al., 1978; Vanderleyden et al., 1979; Laties, 1982). The present work was done to further characterize the oxidative and phosphorylative properties of mitochondria from $N$. crassa harvested in the exponential phase of growth in the presence of chloramphenicol, and to determine the sensitivity to inhibitors of the cytochrome chain and of the alternative oxidase.

Like mitochondria from higher plants (Palmer \& Møller, 1982), mitochondria from $N$. crassa appear to possess three distinct NADH dehydrogenases (Schwitzguébel \& Palmer, 1982): (a) an NADH-ubiquinone oxidoreductase (Complex I), sensitive to rotenone and coupled to the formation of ATP; $(b)$ a rotenone-insensitive NADH dehydrogenase, not linked to the phosphorylation, which is very active in the early exponential phase of growth and $(c)$ a dehydrogenase with its catalytic site on the outer surface of the inner mitochondrial membrane, able to oxidize exogenous NADH in a cation-dependent manner (Møller et al., 1982). The possible effect of the treatment that derepressed the cyanide-insensitive oxidase on the activity of these different dehydrogenases was investigated in this work.

$\dagger$ Present address: Laboratory of Plant Physiology, University of Neuchatel, Chantemerle 20, CH-2000 Neuchatel, Switzerland.

Abbreviations: CLAM, $m$-chlorobenzohydroxamic acid; FCCP, carbonyl cyanide $p$-trifluoromethoxyphenylhydrazone; RCR, respiratory control ratio. 
The alternative oxidase could branch from the main respiratory chain at the ubiquinone level. In this situation a single homogeneous pool of quinone would receive electrons from the various dehydrogenases and distribute them to both oxidases. Alternatively, different respiratory systems may be parallel chains with distinct pools of ubiquinone. In mitochondria from Acanthamoeba castellanii, wheat roots, sweet potatoes and cassava, endogenous NADH donates electrons to the cyanide-resistant oxidase, whereas electrons from exogenous NADH have only access to the cyanide-sensitive oxidase (Edwards \& Lloyd, 1978; Palmer, 1979). Moreover, in different plant mitochondria, malic enzyme appears to be connected to the alternative oxidase by a specific pool of $\mathrm{NAD}^{+} / \mathrm{NADH}$ and a rotenone-resistant dehydrogenase, whereas a second pool of $\mathrm{NAD}^{+} / \mathrm{NADH}$ appears to link malate dehydrogenase to the cytochrome chain via a rotenone-sensitive dehydrogenase (Rustin et al., 1980). The relationship between the different rotenone-sensitive and -resistant dehydrogenases and both oxidases was therefore investigated for mitochondria isolated from $N$. crassa grown in the presence of chloramphenicol.

The coexistence of an alternative oxidase with the cytochrome chain suggests the operation of a mechanism able to control the distribution of the electron flux between both pathways. The nature of this regulation remains unknown, although it has been reported that exogenous nucleoside 5 -monophosphates could selectively stimulate the cyanide-insensitive respiration in mitochondria isolated from several micro-organisms, including $N$. crassa (Hanssens \& Verachtert, 1976; Doussière et al., 1979; Vanderleyden et al., 1979, 1980; Ziogas \& Georgopoulos, 1980). Since the inner mitochondrial membrane may not be permeable to AMP, it has been suggested that this nucleotide acts on a compound located on the outer surface of the inner membrane (Vanderleyden et al., 1979). Moreover, the effect of AMP depends on the procedure used to prepare mitochondria, suggesting that AMP may easily be lost from the organelle (Vanderleyden et al., 1979). The possible effect of AMP on the alternative oxidase of $N$. crassa was therefore re-evaluated using highly intact mitochondria.

\section{METHODS}

Strain and growth conditions. Neurospora crassa, wild-type, strain STA4 (FGSC 262) was kindly supplied by the Fungal Genetics Stock Center (Humboldt State University, Arcata, Calif., U.S.A.). The growth medium was derived from the Neurospora minimal medium produced by Difco, with $58 \mathrm{~mm}$-sucrose as the source of carbon and energy. The complete medium (pH 5.7 at $25^{\circ} \mathrm{C}$ ) was sterilized by autoclaving at $120^{\circ} \mathrm{C}$ for $15 \mathrm{~min}$. Chloramphenicol (Boehringer) was added at a final concentration of $10 \mathrm{~mm}$, immediately before starting growth of the fungus.

To grow hyphae in the presence of chloramphenicol ('chloramphenicol' cells), $10 \mathrm{ml}$ of a suspension of conidia ( $A_{610} 5 \cdot 0$ to 6.0) were transferred into 2 litre Erlenmeyer flasks containing $500 \mathrm{ml}$ liquid growth medium. Cultures were grown at $30^{\circ} \mathrm{C}$ on a rotary shaker $(120$ r.p.m.). Hyphae were harvested after $48 \mathrm{~h}$, in the late-exponential phase of growth [ 9 to $11 \mathrm{~g}$ wet wt $\left.(1 \text { culture })^{-1}\right]$. Control experiments were done with hyphae grown in the absence of chloramphenicol ('normal' cells). For these growth conditions, conidia were inoculated at a lower concentration $\left(A_{610} 2 \cdot 5\right.$ to $\left.3 \cdot 0\right)$. Hyphae were also harvested in the late-exponential phase of growth (13 to $15 \mathrm{~g}$ wet wt $\mathrm{l}^{-1}$ ), after $24 \mathrm{~h}$.

Isolation and purification of mitochondria. Mitochondria were isolated from N. crassa hyphae as described by Schwitzguébel \& Palmer (1982).

When needed, mitochondria were purified by centrifugation on Percoll density gradients (Schwitzguébel et al., 1981). After centrifugation, the gradient was collected by pumping from the bottom of the tube. Fractions ( $1 \mathrm{ml})$ were collected and their density was calculated from their refractive index measured at room temperature. The activities of succinate dehydrogenase and $\mathrm{NADH}$ oxidase were measured in the different fractions without further treatment.

Measurements. The rate of oxygen consumption was measured polarographically as previously described (Schwitzguébel \& Palmer, 1982). Concentrations of substrates were: $20 \mathrm{~mm}$-citrate (plus $1 \mathrm{~mm}-\mathrm{L}-\mathrm{malate}$ ), $20 \mathrm{mm-}$ 2-oxoglutarate (plus $1 \mathrm{~mm}$-L-malate), $20 \mathrm{~mm}$-L-malate (plus $5 \mathrm{~mm}$-L-glutamate), $20 \mathrm{~mm}$-succinate, $2 \mathrm{~mm}-\mathrm{NADH}$, $2 \mathrm{~mm}$-NADPH. When required, 0.1 to $0.5 \mathrm{~mm}$-ADP, $0.1 \mathrm{mM}$-AMP, $0.4 \mu \mathrm{M}$-carbonyl cyanide $p$-trifluoromethoxyphenylhydrazone (FCCP), $0.5 \mathrm{~mm}$-atractylate, $20 \mu \mathrm{M}$-rotenone, $10 \mu \mathrm{M}$-antimycin, $1 \mathrm{mM}-\mathrm{KCN}, 1 \mathrm{~mm}-\mathrm{m}$-chlorobenzohydroxamic acid (CLAM), 1 mM-sodium azide were added to the reaction mixture.

Respiratory control ratios (RCR) and $\mathrm{ADP} / \mathrm{O}$ ratios were calculated from oxygen electrode traces by the responses of mitochondria to quantitative additions of ADP, according to Chance \& Williams (1955). 
The activity of the succinate dehydrogenase (EC 1.3.99.1) was measured as previously described (Schwitzguébel et al., 1981).

Protein was measured by the Lowry method using bovine serum albumin as the standard.

\section{RESULTS}

\section{Oxidation of $\mathrm{NAD}^{+}$-linked substrates}

The ability of mitochondria isolated from $N$. crassa to oxidize $\mathrm{NAD}^{+-}$-linked substrates was investigated for hyphae grown in the absence ('normal' cells) or in the presence of chloramphenicol ('chloramphenicol' cells). Both types of mitochondria oxidized citrate at similar rates in State 3, whereas mitochondria from 'chloramphenicol' cells oxidized 2oxoglutarate and malate (plus glutamate) $15 \%$ and $40 \%$ faster, respectively, than mitochondria from 'normal' cells (Table 1). In State 4, the rate of oxidation of malate was almost doubled, whereas those of citrate and 2-oxoglutarate were increased by only $30 \%$. In contrast, the efficiency of the phosphorylation markedly decreased in mitochondria from "chloramphenicol' cells, and the ADP/O ratios were reduced by 0.8 (malate) to 1.2 (citrate or 2-oxoglutarate).

Respiration of mitochondria from 'normal' cells was not affected by the addition of $1 \mathrm{~mm}$ CLAM, but was almost totally inhibited by either $20 \mu \mathrm{M}$-antimycin or $1 \mathrm{mM}$-cyanide (results not shown). In contrast, the oxidation of $\mathrm{NAD}^{+}$-linked substrates by mitochondria from 'chloramphenicol' cells was largely resistant to cyanide (Table 2) or to antimycin (results not shown) in State 3, and totally insensitive to both inhibitors in State 4 (results not shown). The maximal activity of the oxidase resistant to cyanide, higher than the activity sensitive to CLAM, showed a great variability according to the substrate oxidized (Table 2). In contrast, the rates of oxidation resistant to CLAM, which were higher than the rates sensitive to cyanide, were found to be roughly similar for the different substrates. In the absence of any inhibitor, the rates of

Table 1. Effect of growing N. crassa in the presence of chloramphenicol on the mitochondrial oxidation of $\mathrm{NAD}^{+}$-linked substrates

Mitochondria were isolated from $N$. crassa grown in the absence or the presence of chloramphenicol. Oxygen uptake $\left[\mathrm{nmol} \mathrm{O} \mathrm{min}^{-1}\left(\mathrm{mg}\right.\right.$ protein) $\left.{ }^{-1}\right]$ in the absence (State 4) or the presence of ADP (State 3) is expressed as mean value \pm S.E. (no. of determinations in parentheses). The substrates were at the concentrations given in Methods, with 0.4 to $0.7 \mathrm{mg}$ mitochondrial protein.

$$
\text { Substrate State } 3 \text { rate State } 4 \text { rate RCR ADP/O }
$$

(a) Mitochondria from 'normal' cells

\begin{tabular}{|c|c|c|c|c|}
\hline Citrate & $171 \pm 6(5)$ & $47 \pm 2(5)$ & $3.6 \pm 0.2(5)$ & $2 \cdot 5 \pm 0 \cdot 2(5)$ \\
\hline 2-Oxoglutarate & $180 \pm 13(4)$ & $23 \pm 2(4)$ & $7 \cdot 8 \pm 0.4(4)$ & $2 \cdot 5 \pm 0 \cdot 2(4)$ \\
\hline Malate & $110 \pm 7(4)$ & $42 \pm 4(4)$ & $2 \cdot 6 \pm 0 \cdot 3(4)$ & $1 \cdot 9 \pm 0 \cdot 2(4)$ \\
\hline & \multicolumn{4}{|c|}{ (b) Mitochondria from 'chloramphenicol' cells } \\
\hline Citr: & $15(7)$ & $60 \pm 8$ & $8 \pm 0.3$ & $0.1(7)$ \\
\hline 2-Oxoglutara & $207 \pm 13(5)$ & $30 \pm 4(5)$ & $6.9 \pm 0.7(5)$ & $1 \cdot 3 \pm 0 \cdot 2(5)$ \\
\hline Malate & $155 \pm 14(6)$ & $77 \pm 8(6)$ & $2 \cdot 0 \pm 0 \cdot 2(6)$ & $1 \cdot 1 \pm 0.2(6)$ \\
\hline
\end{tabular}

Table 2. Effect of $K C N$ and $C L A M$ on the oxidation of NAD+-linked substrates by mitochondria from 'chloramphenicol' cells

Oxygen uptake in State $3\left[\mathrm{nmol} \mathrm{O} \mathrm{min}^{-1}(\mathrm{mg} \text { protein })^{-1}\right]$ is expressed as mean value \pm S.E. (no. of determinations in parentheses). The substrates were at the concentrations given in Methods, with $0 \cdot 4$ to $0.7 \mathrm{mg}$ of mitochondrial protein.

\begin{tabular}{lcccc}
\multicolumn{1}{c}{ Substrate } & Control & $+\mathrm{KCN}$ & +CLAM & + KCN + CLAM \\
Citrate & $170 \pm 15(7)$ & $117 \pm 12(5)$ & $119 \pm 15(7)$ & $41 \pm 2(3)$ \\
2-Oxoglutarate & $207 \pm 13(5)$ & $156 \pm 7(2)$ & $111 \pm 19(4)$ & $61 \pm 5(3)$ \\
Malate & $155 \pm 14(6)$ & $88 \pm 6(4)$ & $99 \pm 8(6)$ & $44 \pm 4(5)$
\end{tabular}


oxidation were less than would be predicted from the sum of the cyanide-resistant and CLAMresistant rates measured separately, indicating that one, or both, of the oxidases did not operate at maximal activity. In the presence of both cyanide and CLAM, a significant rate of oxygen uptake was still observed. This residual rate was not reduced by further addition of either CLAM, cyanide or antimycin, and was only slightly inhibited after the addition of 1 mM-sodium azide.

The efficiency of the phosphorylation was reduced by the addition of cyanide or antimycin and $\mathrm{ADP} / \mathrm{O}$ ratios of 0.5 to 0.7 were observed, indicating that the first energy-coupling site was only partially active. In contrast, the formation of ATP increased in the presence of CLAM, but the ADP/O ratios (1.5 to 1.7$)$ were much lower than expected if all three energy-coupling sites were fully active, suggesting the possible operation of a rotenone-resistant non-phosphorylating pathway.

Oxidation of $\mathrm{NAD}^{+}$-linked substrates by mitochondria from 'normal' cells was very sensitive to the inhibition by rotenone, although a small amount of rotenone-resistant oxygen consumption was observed (Table 3). The rotenone-sensitive oxidation of $\mathrm{NAD}^{+}$-linked substrates was markedly reduced in mitochondria from 'chloramphenicol' cells (Table 3), whereas the activity of the rotenone-resistant NADH dehydrogenase was strongly enhanced. The rotenone-resistant pathway was not stimulated by the addition of $\mathrm{NAD}^{+}$to mitochondria from 'chloramphenicol' cells (results not shown).

The rotenone-resistant NADH dehydrogenase appears to be able to donate electrons to both cyanide-sensitive and -resistant oxidases, indicating the existence of branched, rather than parallel, respiratory pathways. The activity of the pathway resistant to both rotenone and cyanide showed a great variability depending on the substrate oxidized (Table 4). In contrast, in the presence of both rotenone and CLAM, the rates of oxygen uptake were approximately the same for all $\mathrm{NAD}^{+}$-linked substrates. The ADP/O ratios measured in the presence of rotenone were found to be 0.9 to 1.1 (results not shown), indicating the simultaneous operation of the cytochrome chain and of the alternative oxidase.

\title{
Oxidation of succinate
}

Growth of $N$. crassa in the presence of chloramphenicol markedly enhanced the oxidation of succinate by isolated mitochondria, the increase being more pronounced in State 4 than in State 3 (Table 5). In contrast, the efficiency of the phosphorylation decreased, indicating that both oxidases operated under State 3 conditions. In the absence of added inhibitor, the rate of oxidation was less than would be predicted from the sum of the cyanide- and CLAM-resistant rates, suggesting that one, or both, of the oxidase systems did not function at maximal activity.

Table 3. Effect of growing $N$. crassa in the presence of chloramphenicol on the sensitivity of isolated mitochondria to rotenone

\begin{abstract}
Mitochondria were isolated from $N$. crassa grown in the absence or the presence of chloramphenicol. Oxygen uptake in State $3\left[\mathrm{nmol} \mathrm{O} \mathrm{min}^{-1}(\mathrm{mg} \text { protein })^{-1}\right]$ is expressed as the mean value \pm S.E. (no. of determinations in parentheses). The substrates were at the concentrations given in Methods, with 0.4 to $0.7 \mathrm{mg}$ mitochondrial protein. The rotenone-sensitive rate is defined as the difference between the State 3 rate in the absence of rotenone (Table 1) and the rate of oxidation measured in the presence of rotenone.
\end{abstract}

$\begin{array}{ccc}\text { Rotenone-sensitive } & \text { Rotenone-resistant } \\ \text { Substrate } & \text { rate } & \text { rate }\end{array}$

(a) Mitochondria from 'normal' cells

$\begin{array}{lcl}\text { Citrate } & 145 \pm 14(6) & 26 \pm 2(6) \\ \text { 2-Oxoglutarate } & 152 \pm 14(4) & 28 \pm 3(4) \\ \text { Malate } & 78 \pm 7(4) & 32 \pm 4(4)\end{array}$

(b) Mitochondria from 'chloramphenicol' cells

$\begin{array}{llr}\text { Citrate } & 76 \pm 6(7) & 94 \pm 8(7) \\ \text { 2-Oxoglutarate } & 50 \pm 6(3) & 157 \pm 10(3) \\ \text { Malate } & 59 \pm 7(5) & 96 \pm 9(5)\end{array}$


Table 4. Effect of $K C N$ and CLAM on the rotenone-resistant oxidation of $N A D^{+-l i n k e d}$ substrates by mitochondria from 'chloramphenicol' cells

Oxygen uptake in the presence of both ADP and rotenone $\left[\mathrm{nmol} \mathrm{O}_{2} \mathrm{~min}^{-1}(\mathrm{mg} \text { protein })^{-1}\right]$ is expressed as mean value \pm S.E. (no. of determinations in parentheses). The substrates were at the concentrations given in Methods with 0.4 to $0.7 \mathrm{mg}$ mitochondrial protein.

\begin{tabular}{lcccc}
\multicolumn{1}{c}{ Substrate } & Control & $+\mathrm{KCN}$ & + CLAM & + KCN + CLAM \\
Citrate & $94 \pm 8(7)$ & $72 \pm 11(4)$ & $84 \pm 6(5)$ & $32 \pm 2(4)$ \\
2-Oxoglutarate & $157 \pm 10(3)$ & $113 \pm 13(3)$ & $92 \pm 12(4)$ & $33 \pm 2(2)$ \\
Malate & $96 \pm 9(5)$ & $42 \pm 4(3)$ & $83 \pm 3(4)$ & $31 \pm 2(3)$
\end{tabular}

\title{
Table 5. Effect of growing $N$. crassa in the presence of chloramphenicol on the mitochondrial oxidation of succinate
}

\begin{abstract}
Mitochondria were isolated from $N$. crassa grown in the absence or the presence of chloramphenicol. Oxygen uptake $\left[\mathrm{nmol} \mathrm{O} \mathrm{min}^{-1}\left(\mathrm{mg}\right.\right.$ protein) $\left.{ }^{-1}\right]$ in the absence (State 4) or the presence of ADP (State 3) is expressed as mean value \pm S.E. (no. of determinations in parentheses). The substrates were at the concentrations given in Methods, with 0.2 to $0.4 \mathrm{mg}$ of mitochondrial protein.
\end{abstract}

\begin{tabular}{|c|c|c|c|c|}
\hline Conditions & State 3 rate & State 4 rate & RCR & $\mathrm{ADP} / \mathrm{O}$ \\
\hline & & (a) Mitochon & from 'normal' & \\
\hline ontrol & $220 \pm 12(4)$ & $111 \pm 9(4)$ & $2 \cdot 0 \pm 0 \cdot 1$ & $1 \cdot 4 \pm 0 \cdot 1$ \\
\hline & 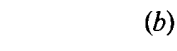 & Aitochondria & 'chloramphen & cells \\
\hline nnt & $427 \pm 39(6)$ & $295 \pm$ & $1.6 \pm$ & $0.6 \pm 0.1(6)$ \\
\hline & $282 \pm 34(5)$ & $268 \pm 22$ & $1 \cdot 1 \pm$ & \\
\hline + CLAM & $236 \pm 10(5)$ & $115 \pm 9(5)$ & $1.9 \pm 0.1$ & $1.4 \pm 0.1(5)$ \\
\hline$+\mathrm{KCN}+\mathrm{CLAM}$ & $87 \pm 6(5)$ & $78 \pm 4(5)$ & $1.1 \pm 0.1$ & - \\
\hline
\end{tabular}

While the State 4 rate remained almost totally unaffected by the addition of cyanide, the rate of oxidation of succinate in State 3 was decreased to the level of State 4. ADP stimulated the rate via the cytochrome chain, and therefore the total respiration rate, whereas it had little influence, if any, on the activity of the alternative oxidase.

The addition of CLAM decreased the rate of oxidation of succinate and enhanced the ADP/O ratio to the value observed with mitochondria from 'normal' cells in the absence of any inhibitor. In the presence of an hydroxamic acid, mitochondria from 'chloramphenicol' cells thus appear to oxidize succinate via the cytochrome chain only. However, in the presence of both CLAM and cyanide, a residual rate of oxygen uptake was observed, not inhibited by further addition of either CLAM, antimycin, cyanide or azide.

\section{Oxidation of exogenous $N A D H$ and $N A D P H$}

Growth of $N$. crassa in the presence of chloramphenicol not only enhanced the activity of the internal NADH dehydrogenase resistant to rotenone, but also strongly increased the ability of mitochondria to oxidize exogenous NADH (fivefold) and NADPH (tenfold) (Table 6). Under State 3 conditions, the respiration of both substrates was largely insensitive to cyanide (Table 6) and antimycin (results not shown), whereas the oxidation rates in State 4 were almost totally resistant to both inhibitors. Furthermore, the low ADP/O ratios observed also indicate that the strong oxidation of $\mathrm{NAD}(\mathrm{P}) \mathrm{H}$ was poorly coupled to the phosphorylation.

In the absence of any added inhibitor, the rate of oxidation of NADH in State 3 was only slightly lower than the sum of the rate resistant to cyanide and of the rate resistant to CLAM, indicating that both systems operated at nearly maximal activity.

The addition of CLAM increased the ADP/O ratio values from 0.4 to 0.8 only, whereas a value compatible with the operation of two energy-coupling sites would be expected. Such a value (1.4) was reached when succinate was oxidized in the presence of CLAM (Table 5). When 
Table 6. Effect of growing $N$. crassa in the presence of chloramphenicol on the mitochondrial oxidation of exogenous $N A D H$ and $N A D P H$

\begin{abstract}
Mitochondria were isolated from $N$. crassa grown in the absence or presence of chloramphenicol. Oxygen uptake $\left[\mathrm{nmol} \mathrm{O} \mathrm{min}^{-1}\right.$ (mg protein) $\left.{ }^{-1}\right]$ in the absence (State 4) or the presence of ADP (State 3) is expressed as mean value \pm S.E. (no. of determinations in parentheses). The substrates were at the concentrations given in Methods, with 0.1 to $0.2 \mathrm{mg}$ of mitochondrial protein.
\end{abstract}

$\begin{array}{llll}\text { Substrate } & \text { State } 3 \text { rate } & \text { State } 4 \text { rate } & \text { RCR } / O\end{array}$

(a) Mitochondria from 'normal' cells

$\begin{array}{lclll}\text { NADH } & 198 \pm 16(5) & 66 \pm 8(5) & 3.0 \pm 0.2(5) & 1.5 \pm 0.1(5) \\ \text { NADPH } & 64 \pm 3(4) & 44 \pm 4(4) & 1.5 \pm 0.2(4) & 1.6 \pm 0.1(4)\end{array}$

(b) Mitochondria from 'chloramphenicol' cells

$\begin{array}{lrllc}\text { NADH } & 1086 \pm 94(6) & 675 \pm 58(6) & 1 \cdot 8 \pm 0 \cdot 2(6) & 0 \cdot 4 \pm 0 \cdot 1(6) \\ + \text { KCN } & 833 \pm 66(4) & 618 \pm 60(4) & 1 \cdot 4 \pm 0 \cdot 2(4) & - \\ + \text { CLAM } & 314 \pm 23(4) & 129 \pm 26(4) & 2 \cdot 0 \pm 0 \cdot 2(4) & 0 \cdot 8 \pm 0 \cdot 1(4) \\ + \text { KCN + CLAM } & 145 \pm 15(4) & 104 \pm 11(4) & 1 \cdot 4 \pm 0 \cdot 2(4) & - \\ \text { NADPH } & 624 \pm 54(5) & 444 \pm 36(5) & 1 \cdot 6 \pm 0 \cdot 2(5) & 0 \cdot 4 \pm 0 \cdot 1(5) \\ + \text { KCN } & 519 \pm 49(4) & 393 \pm 39(4) & 1 \cdot 4 \pm 0 \cdot 2(4) & - \\ + \text { CLAM } & 222 \pm 15(4) & 100 \pm 7(4) & 2 \cdot 2 \pm 0 \cdot 2(4) & 0 \cdot 7 \pm 0 \cdot 1(4) \\ + \text { KCN + CLAM } & 131 \pm 12(4) & 105 \pm 6(4) & 1 \cdot 3 \pm 0 \cdot 1(4) & \end{array}$

the alternative oxidase was inhibited, part of the electron flux resulting from the oxidation of exogenous NAD(P)H was therefore probably not passing through the cytochrome chain. Furthermore, in the presence of both cyanide (or antimycin) and CLAM, the rates of oxidation of $\mathrm{NAD}(\mathrm{P}) \mathrm{H}$ remained above $100 \mathrm{nmol} \mathrm{O}_{2} \min ^{-1}(\mathrm{mg} \text { protein })^{-1}$ and were not significantly inhibited by the addition of $1 \mathrm{~mm}$-azide.

Centrifugation of the washed mitochondrial pellet on a Percoll density gradient revealed that the total and the cyanide-resistant NADH oxidase activities, as measured in the presence of an uncoupler $(0 \cdot 4 \mu \mathrm{M}$-FCCP), were found in the same fractions as succinate dehydrogenase (Fig. 1$)$. The very high cyanide-resistant oxidation of NADH was therefore clearly linked to mitochondria and was not due to an increased contamination by other sub-cellular organelles.

As determined by centrifugation on a Percoll gradient, the buoyant density of mitochondria from 'chloramphenicol' cells was found to be between 1.071 and 1.075 (Fig. 1). A second small peak of mitochondrial activities was found at a density of $1 \cdot 060$. No significant difference in the sensitivity to either CLAM or cyanide was found between both populations of mitochondria, as tested for the respiration of several substrates (results not shown).

\title{
Effect of AMP on the alternative oxidase
}

The addition of $0.1 \mathrm{~mm}$-AMP clearly stimulated the cyanide-insensitive oxidation of citrate by mitochondria isolated from $N$. crassa grown in the presence of chloramphenicol, and the increased respiration was inhibited by CLAM (Fig. 2, trace $b$ ). However, AMP failed to activate significantly the alternative oxidase in the presence of either FCCP, an uncoupler, or atractylate, an inhibitor of the translocation of ADP across the inner mitochondrial membrane (Fig. 2, traces $c$ and $d$ ). Furthermore, when the alternative oxidase was inhibited by CLAM, AMP still stimulated the oxygen uptake and respiratory control ratios could be measured (Fig. 2, trace $a$ ).

A small inhibition of the alternative oxidase was even observed after the addition of AMP to mitochondria oxidizing succinate (Fig. 3, trace $a$ ). In contrast, AMP stimulated the CLAMresistant rate of oxidation of succinate for a limited period of time, followed by a State 3 to State 4 transition (Fig. 3, trace b). This stimulation was not observed in the presence of atractylate (Fig. 3, trace $c$ ).

The cyanide-resistant oxidation of exogenous NADH was slightly stimulated by AMP (Fig. 3, trace $d$ ), but the cyanide-sensitive pathway was also increased (Fig. 3, trace $f$ ). The effect of AMP on both cyanide-sensitive and -resistant rates of oxidation was however abolished by FCCP (Fig. 3, traces $e$ and $g$ ). 


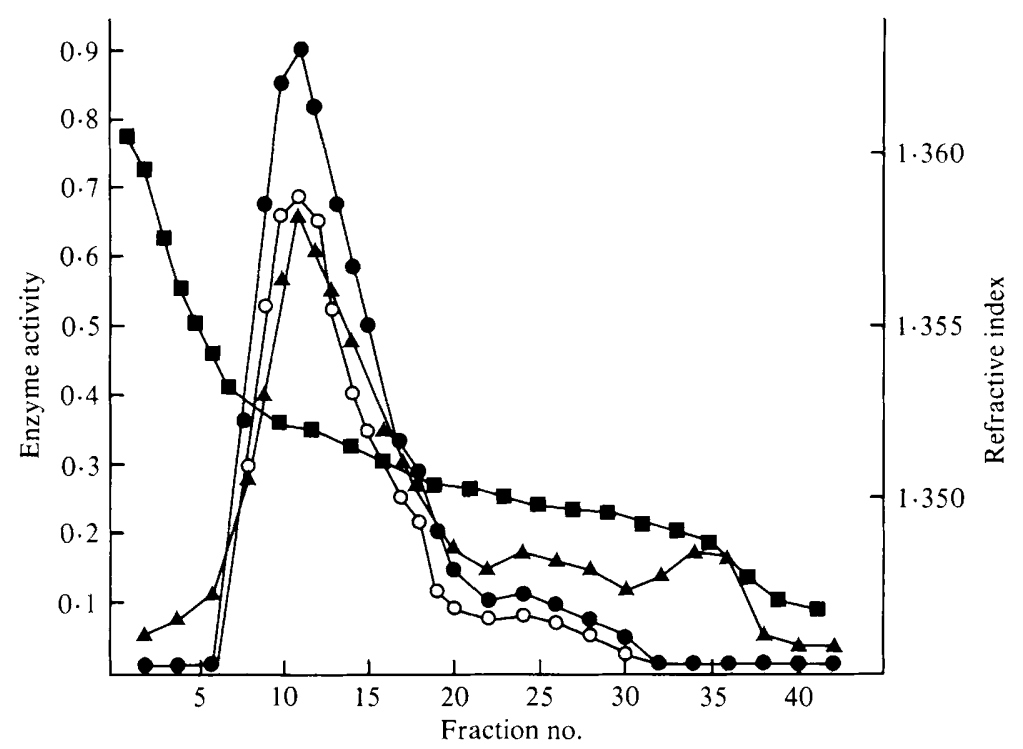

Fig. 1. Percoll density gradient centrifugation of a mitochondrial pellet ( $15 \mathrm{mg}$ protein) isolated from $N$. crassa grown in the presence of chloramphenicol. Enzyme activities are expressed as units per fraction $(1 \mathrm{ml})$. A unit is defined as $1 \mu \mathrm{mol} 2,6$-dichlorophenolindophenol reduced $\mathrm{min}^{-1}$ for succinate dehydrogenase $(\boldsymbol{\Delta})$, and as $1 \mu \mathrm{mol} \mathrm{O}_{2}$ consumed $\min ^{-1}$ for $\mathrm{NADH}$ oxidase. NADH oxidase was measured in the presence of $0.4 \mu \mathrm{M}-\mathrm{FCCP}$, with $(\mathrm{O})$ or without $(\mathrm{O}) 1 \mathrm{~mm}-\mathrm{KCN}$. The refractive index was measured at room temperature

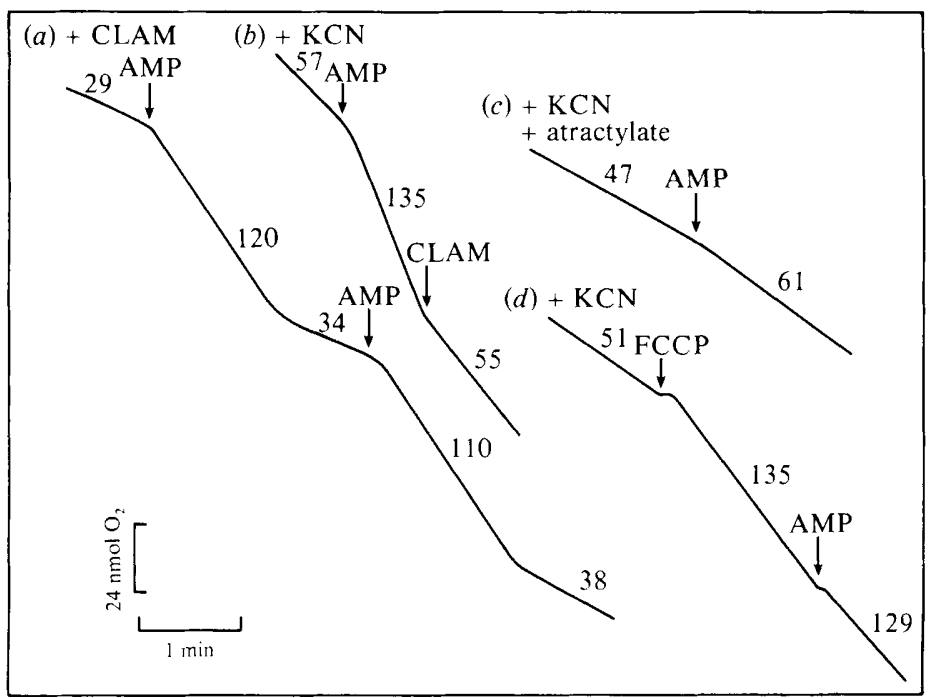

Fig. 2. Effect of exogenous AMP on the oxidation of citrate by mitochondria isolated from $N$. crassa grown in the presence of chloramphenicol. Oxygen uptake was measured as described in Methods, with 0.5 (trace $a$ ) 0.65 (trace $b$ ) or 0.4 (traces $c$ and $d$ ) mg mitochondrial protein. Numbers above the traces represent the rates of oxidation expressed in nmol $\mathrm{O}_{2} \mathrm{~min}^{-1}(\mathrm{mg} \text { protein })^{-1}$.

As determined by the release of $\mathrm{NAD}^{+}$-linked isocitrate dehydrogenase after the addition of Triton X-100 (Schwitzguebel \& Palmer, 1982), the integrity of the inner membrane of mitochondria isolated from 'chloramphenicol' cells was shown to be approximately $96 \%$. The results presented (Figs 2 and 3) clearly indicate that AMP did not selectively stimulate the cyanide-insensitive respiration of such intact mitochondria. The apparent stimulation of the 


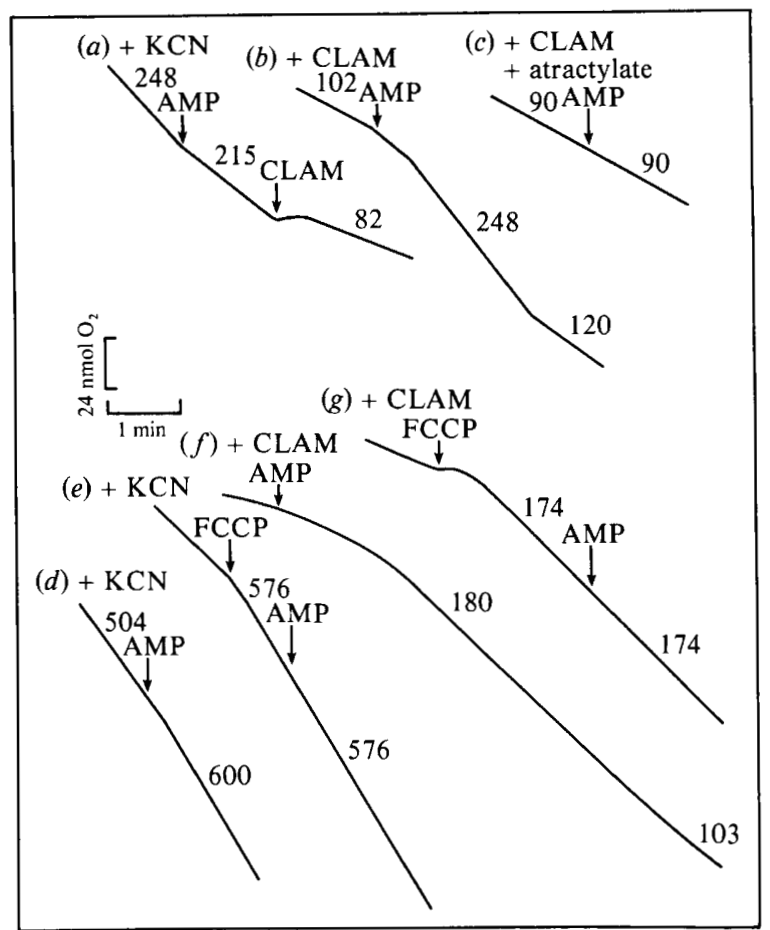

Fig. 3. Effect of exogenous AMP on the oxidation of succinate and NADH by mitochondria isolated from $N$. crassa grown in the presence of chloramphenicol. Oxygen uptake was measured as described in Methods, with 0.3 to 0.5 (traces $a$ to $c$ ) or 0.2 to 0.25 (traces $d$ to $g$ ) mg mitochondrial protein. Numbers above the traces represent the rates of oxidation as expressed in $\mathrm{nmol} \mathrm{O}_{2} \mathrm{~min}^{-1}$ (mg protein) ${ }^{-1}$.

rotenone- and cyanide-sensitive pathway was probably due to the ADP produced from exogenous AMP and endogenous ATP by the adenylate kinase present in the intermembrane space of $N$. crassa mitochondria (Henry et al., 1980). The possible effect of other nucleoside 5'monophosphates on the alternative oxidase was therefore not further investigated.

\section{DISCUSSION}

Results presented here confirm that the respiration via the cytochrome chain is decreased and the activity of the cyanide-insensitive oxidase is derepressed when $N$. crassa is grown in the presence of chloramphenicol. The activity of the cyanide-resistant, CLAM-sensitive oxidase was clearly present in mitochondria purified by centrifugation on a Percoll density gradient and was not due to an increased contamination by other sub-cellular organelles. These results are in contrast to the observation that cyanide-resistant respiration in yeast seems to be associated with heterogeneous membraneous particles apparently non mitochondrial in origin (Ainsworth et al., 1980). Moreover, gradient-purified mitochondria from wheat seedlings lack cyanideinsensitive respiration, whereas the apparent activity of the alternative oxidase in crude mitochondrial pellets was due to contamination by extramitochondrial lipoxygenase (Goldstein et al., 1981). Results obtained with exogenous NADH as a substrate suggest that the maximal activities of the cytochrome chain and of the alternative oxidase could be 300 to 350 and 800 to $900 \mathrm{nmol} \mathrm{O}_{2} \mathrm{~min}^{-1}$ (mg protein) $)^{-1}$, respectively, for mitochondria isolated from $N$. crassa grown in the presence of chloramphenicol.

Results presented in this paper clearly indicate that the addition of exogenous AMP to isolated mitochondria did not specifically stimulate the electron flux through the alternative 
oxidase. This means that AMP possibly present in the inner mitochondrial membrane in vivo was not lost in our mitochondrial preparations and its concentration was high enough to fully activate the alternative oxidase. Alternatively, AMP did not stimulate at all the cyanideinsensitive oxidase of mitochondria from $N$. crassa, since even the concept of the activation of a non-phosphorylating pathway by low energy compounds such as nucleoside monophosphates is somewhat questionable. In such a case, however, some artefact could be observed if the mitochondrial inner membrane is damaged during the isolation procedure.

Growth of $N$. crassa in the presence of chloramphenicol not only hindered the respiration via the cytochrome chain and derepressed the activity of the alternative oxidase, but it also had important effects on the different NADH dehydrogenases present in the inner mitochondrial membrane.

The oxidation of $\mathrm{NAD}^{+}$-linked substrates by mitochondria isolated from $N$. crassa grown in the presence of chloramphenicol became largely insensitive to rotenone. Apparently, the biogenesis of a functional, rotenone-sensitive, NADH-ubiquinone oxidoreductase (Complex I) was hindered by chloramphenicol. Although further studies will be required to confirm this assumption, we suggest that at least one sub-unit of Complex I could be synthesized on mitochondrial ribosomes. NADH-ubiquinone oxidoreductase isolated from beef heart was reported to be a complex structure of at least 26 different polypeptides (Heron et al., 1979), whereas the polypeptide composition of Complex I of mitochondria from $N$. crassa remains unknown. Like other complexes present in the inner mitochondrial membrane (cytochrome $b c_{1}$, cytochrome oxidase, ATPase), Complex I could also be synthesized partly on mitochondrial and partly on cytoplasmic ribosomes.

In contrast, the activity of the internal, rotenone-resistant NADH dehydrogenase, normally high in the early-exponential phase of growth (Schwitzguébel \& Palmer, 1982), was markedly increased by growing $N$. crassa in the presence of chloramphenicol. The rotenone-sensitive and -resistant oxidation of NADH could be due to the existence of two distinct dehydrogenases or to the presence of two active sites on the same protein. Complex I from rat liver mitochondria reduces ubiquinone analogues by two pathways, one sensitive and the other resistant to rotenone (Di Virgilio \& Azzone, 1982). It is therefore possible that chloramphenicol hindered the biosynthesis of the rotenone-sensitive site without decreasing the activity of the rotenoneresistant site of Complex I. Such a situation has been described for respiration-defective chinese hamster cell mutants containing low levels of NADH-ubiquinone oxidoreductase sensitive to rotenone (Malczewski \& Whitfield, 1982).

Finally, the ability of mitochondria to oxidize exogenous NADH and NADPH was strongly enhanced in $N$. crassa grown in the presence of chloramphenicol. A very high rate of reoxidation of cytosolic reduced coenzymes by the mitochondria would enable an increased flux through glycolysis and the hexose monophosphate shunt, favour ATP formation in the cytosol and therefore compensate the deficiency of the mitochondrial oxidative phosphorylation.

The different dehydrogenases appear to be able to give electrons to both cyanide-sensitive and -resistant oxidases, indicating the operation of branched (Von Jagow \& Bohrer, 1975) rather than parallel respiratory pathways (Edwards \& Lloyd, 1978; Rustin et al., 1980). There is no evidence for a complete nonphosphorylating pathway, parallel to the energy-conserving respiratory chain.

Since the oxidation of the different substrates was not totally inhibited by the addition of both cyanide (or antimycin) and CLAM, the results presented here did not allow us to estimate if the cytochrome chain was always functioning at its maximal capacity (model of Bahr \& Bonner, 1973), or if electrons were randomly distributed between both oxidases (model of De Troostembergh \& Nyns, 1978).

The possible existence of a second alternative oxidase, insensitive to both cyanide and hydroxamic acids, has been reported for Paramecium tetraurelia (Doussière et al., 1979), Ustilago maydis (Ziogas \& Georgopoulos, 1980) and N. crassa, strain inl-89601, grown in the presence of chloramphenicol (Edwards \& Unger, 1978). However, this third pathway, apparently sensitive to azide, is only poorly associated with mitochondria isolated from N. crassa (Edwards \& Unger, 1978). Furthermore, we did not observe any significant azide-sensitive respiration in 
mitochondria from wild-type $N$. crassa grown in the presence of chloramphenicol. Such results make somewhat questionable the existence of a cyanide-insensitive, azide-sensitive oxidase in mitochondria from $N$. crassa. It is therefore likely that the oxygen uptake observed in the presence of both cyanide and CLAM was only an artefact due to the auto-oxidation of some very reduced intermediate, although the use of other inhibitors (e.g. sulphide) might reveal a further pattern of specific inhibition comparable with that observed in Tetrahymena pyriformis (Lloyd et $a l ., 1980)$ where cyanide-insensitive but sulphide-sensitive respiration has been shown.

This work was supported by the Swiss National Science Foundation (grant no. 83.615.0.78 to J.P.S.) and the Science Research Council.

\section{REFERENCES}

Ainsworth, P. J., Ball, A. J. S. \& Tustanoff, E. R. (1980). Cyanide-resistant respiration in yeast. I. Isolation of a cyanide-insensitive $\mathrm{NAD}(\mathrm{P}) \mathrm{H}$ oxidoreductase. Archives of Biochemistry and Biophysics 202, 172-186

Bahr, J. T. \& Bonner, W. D. (1973). Cyanide insensitive respiration. II. Control of the alternate pathway. Journal of Biological Chemistry 248, 34463450 .

Chance, B. \& Williams, G. R. (1955). Respiratory enzymes in oxidative phosphorylation. I. Kinetics of oxygen utilization. Journal of Biological Chemistry 217, 383-393.

De Troostembergh, J. C. \& Nyns, E. J. (1978). Kinetics of the respiration of cyanide-insensitive mitochondria from the yeast Saccharomycopsis lipolytica. European Journal of Biochemistry 85, 423432.

Di Virgilio, F. \& Azzone, G. F. (1982). Activation of site I redox-driven $\mathrm{H}^{+}$pump by exogenous quinones in intact mitochondria. Journal of Biological Chemistry 257, 4106-4113.

Doussière, J., Sainsard-Chanet, A. \& Vignais, P. V. (1979). The respiratory chain of Paramecium tetraurelia in wild type and the mutant $\mathrm{Cl}_{1}$. II. Cyanideinsensitive respiration. Function and regulation. Biochimica et biophysica acta 548, 236-252.

Edwards, D. L. \& Unger, B. W. (1978). Cyanide- and hydroxamate-resistant respiration in Neurospora crassa. Journal of Bacteriology 133, 1130-1134.

Edwards, S. W. \& Lloyd, D. (1978). Properties of mitochondria isolated from cyanide-sensitive and cyanide-stimulated cultures of Acanthamoeba castellanii. Biochemical Journal 174, 203-211.

Goldstein, A. H., Anderson, J. E. \& McDaniel, R. G. (1981). Cyanide-insensitive and cyanidesensitive $\mathrm{O}_{2}$ uptake in wheat. II. Gradient-purified mitochondria lack cyanide-insensitive respiration. Plant Physiology 67, 594-596.

Hanssens, L. \& Verachtert, H. (1976). Adenosine 5'monophosphate-stimulated cyanide-insensitive respiration in mitochondria of Moniliella tomentosa. Journal of Bacteriology 125, 829-836.

HenRy, L. E. A., Cammack, R., SChwitzguébel, J. P., PALMER, J. M. \& Hall, D. O. (1980). Intracellular localization, isolation and characterization of two distinct varieties of superoxide dismutase from Neurospora crassa. Biochemical Journal 187, 321-328.

Heron, C., Smith, S. \& Ragan, C. I. (1979). An analysis of the polypeptide composition of bovine heart mitochondrial NADH-ubiquinone oxidoreductase by two-dimensional polyacrylamide-gel electrophoresis. Biochemical Journal 181, 435-443.

lambowitz, A. M., Slayman, C. W., Slayman, C. L. \& BONNER, W. D. (1972a). The electron transport components of wild type and poky strains of Neurospora crassa. Journal of Biological Chemistry 247, 1536-1545.

Lambowitz, A. M., Smith, E. W. \& Slayman, C. W. (1972b). Electron transport in Neurospora mitochondria. Journal of Biological Chemistry 247, 4850-4858.

Lambowitz, A. M., Smith, E. W. \& Slayman, C. W. $(1972 c)$. Oxidative phosphorylation in Neurospora mitochondria. Journal of Biological Chemistry 247, 4859-4865.

La Polla, R. J. \& Lambowitz, A. M. (1977). Mitochondrial ribosome assembly in Neurospora crassa. Journal of Molecular Biology 116, 189-205.

LATIES, G. G. (1982). The cyanide-resistant, alternative path in higher plant respiration. Annual Review of Plant Physiology 33, 519-555.

Lloyd, D., Kristensen, B. \& Degn, H. (1980). The effect of inhibitors on the oxygen kinetics of terminal oxidases of Tetrahymena pyriformis ST. Journal of General Microbiology 121, 117-125.

MalczeWsKi, R. M. \& Whitfield, C. D. (1982). Respiration-defective chinese hamster cell mutants containing low levels of NADH-ubiquinone reductase and cytochrome $c$ oxidase. Journal of Biological Chemistry 257, 8137-8142.

Møller, I. M., Schwitzguébel, J. P. \& Palmer, J. M. (1982). Binding and screening by cations and the effect on exogenous $\mathrm{NAD}(\mathrm{P}) \mathrm{H}$ oxidation in Neurospora crassa mitochondria. European Journal of Biochemistry 123, 81-88.

Palmer, J. M. (1979). The 'uniqueness' of plant mitochondria. Biochemical Society Transactions 7 , 246-252.

Palmer, J. M. \& Møller, I. M. (1982). Regulation of NAD(P)H dehydrogenases in plant mitochondria. Trends in Biochemical Sciences 7, 258-261.

Rustin, P., Moreau, F. \& Lance, C. (1980). Malate oxidation in plant mitochondria via malic enzyme and the cyanide-insensitive electron transport pathway. Plant Physiology 66, 457-462.

Schwitzgú́bel, J. P. \& Palmer, J. M. (1982). Properties of mitochondria as a function of the growth stages of Neurospora crassa. Journal of Bacteriology 149, 612-619.

Schwitzguébel, J. P., Møller, I. M. \& Palmer, 
J. M. (1981). Changes in density of mitochondria and glyoxysomes from Neurospora crassa: a reevaluation utilizing silica sol gradient centrifugation. Journal of General Microbiology 126, 289-295.

Shepherd, M. G., Chin, C. M. \& Sullivan, P. A (1978). The alternate respiratory pathway of Candida albicans. Archives of Microbiology 116, 61-67.

Slayman, C. W. (1978). The function of an alternative terminal oxidase in Neurospora. In Functions of Alternative Terminal Oxidases, p. 159. Edited by $\mathrm{H}$. Degn, D. Lloyd \& G. C. Hill. Oxford: Pergamon Press.

VANDERleyden, J., Kurth, J, \& Verachtert, $H$. (1979). Characterization of cyanide-insensitive respiration in mitochondria and sub-mitochondrial particles of Moniliella tomentosa. Biochemical Journal 182, 437-443.

Vanderleyden, J., Peeters, C., Verachtert, H. \& BERTRAND, H. (1980). Stimulation of the alternative oxidase of Neurospora crassa by nucleoside phosphates. Biochemical Journal 188, 141-144.

VON JAGOW, G. \& BOHRER, C. (1975). Inhibition of electron transfer from ferro-cytochrome $b$ to ubiquinone, cytochrome $c_{1}$ and duroquinone by antimycin. Biochimica et biophysica acta 387, 409-424.

Ziogas, B. N. \& Georgopoulos, S. G. (1980). Chloramphenicol-induction of a second cyanideand azide-insensitive mitochondrial pathway in Ustilago maydis. Biochimica et biophysica acta $\mathbf{5 9 2}$, 223-234. 